\title{
Consensus Development Conference: Promoting Access to Quality Palliative Care in Canada
}

\author{
Konrad Fassbender, MA, PhD ${ }^{1,2}$
}

\begin{abstract}
Objective: This article outlines the development and implementation of a consensus development conference (CDC). Background: As a rapid method for data synthesis, a CDC affords a timely and methodic means of evaluating data to effect change in healthcare policy.

Methods: The CDC methodology was adopted for the Palliative Care Matters initiative due to its engagement with the public, scientific community, and palliative care stakeholders.

Results: It requires the involvement of seven key groups/roles to successfully effect change: a manager, steering committee, scientific expert panel, public lay panel, a lay-panel facilitator, a public audience, and the media for dissemination.

Discussion: This article also details the background information and guiding principles on which the Palliative Care Matters initiative was formed. A Canadian Reference Working Group was formed to develop the Palliative Care Matters guiding principles into six scientific questions. The scientific articles in this supplemental issue each present evidence and expert recommendations that speak to one of the Palliative Care Matters scientific questions.
\end{abstract}

Keywords: Consensus Development Conference; palliative care; Palliative Care Matters; public engagement

\section{Background}

$\mathbf{T}$ he Covenant Health Palliative Institute posits that a consensus development conference (CDC) constitutes a foundation for a strong public engagement plan. Developed in Denmark and first implemented in 1987, the CDC is a tool intended to bridge the gap between the general public, scientific experts, and politicians. The intended audience for a $\mathrm{CDC}$ is government, administrators, and other decision makers, in addition to the public. A CDC results in a document reflecting the evidence, opinions, and recommendations on an issue. In partnership with Canada's leading healthcare organizations, the Covenant Health Palliative Institute is undertaking a threephase national CDC on palliative care. The objective of the conference is to develop a national consensus statement and recommendations promoting consistent and timely access to high-quality, coordinated, and integrated palliative and end-oflife care. The purpose of this article, and indeed this issue, is to document the key decisions and learnings from this project.

A CDC is a stylized method which includes a rapid data synthesis of evidence. As such, the review is not intended to generate new scientific data and therefore cannot solve or contribute to a solution. ${ }^{1} \mathrm{New}$ interpretations and evaluation of the data, however, are facilitated when evidence is synthesized (in our case, for review by the Canadian public).

"The participants may formulate the best current judgment of a given technology in relation to the healthcare service requirements, the patients' desires, and the demands of the society."

Suitable topics are topical, not too abstract, contain conflicts, call for clarification of objectives and attitudes, depend on expert contribution for clarification, and require availability of knowledge and expertise. The following criteria for the selection of our topic have been proposed ${ }^{2}$ :

"1. The issue should have public importance;

2. There should be controversy over scientific aspects of the issue;

3. There should be available evidence upon which to base evaluation of the issue; and

4. The issue should be amenable to clarification on technical grounds."

Guidance for the clear and unambiguous wording of questions has been established. Careful and precise definition of the questions to be addressed is required to investigate the topic efficiently ${ }^{3}$ to maximize the opportunity for a valid evaluation

\footnotetext{
${ }^{1}$ Covenant Health Palliative Institute, Edmonton, Alberta, Canada.

${ }^{2}$ Department of Oncology, University of Alberta, Edmonton, Alberta, Canada.

Accepted August 22, 2017.
} 
and to avoid a predetermined conclusion. ${ }^{4}$ Poorly worded questions can result in ambiguous or overly generalized recommendations. ${ }^{5}$ The questions will be used by IPSOS and the scientific panel to gather qualitative and quantitative evidence.

\section{Framework}

Excellence within healthcare is typically isolated and unknown to the system. The introduction and evolution of palliative care in Canada can best be described as opportunistic, relying on advocacy from local champions. The result of this patchwork approach is unequal access to palliative care. Performance standards are in their infancy which makes it difficult to evaluate the quality of services available. Standardization of high-quality palliative care first requires identification of best practices, their evidence, and quality measures across organizations. We propose to use the Institute for Healthcare Improvement (IHI) Trip AIM framework to ensure quality is assessed through (1) improving the health of the defined population, (2) enhancing the patient care experience (including quality, access, and reliability), and (3) reducing, or at least controlling, the per capita cost of care. A primary objective of the CDC is to engage the public and identify practices which satisfy these three criteria.

Second, closing the gap of best and usual practice entails a mechanism and process to spread innovative practices. Barriers which inhibit the diffusion of innovative practices include "the characteristics of the innovation itself; the willingness or ability of those making the adoption to try the new ideas; and characteristics of the culture and infrastructure of the organization to support change." In response, IHI initiated a process to facilitate and accelerate change resulting in the development of a framework in $2006 .{ }^{6}$ The process includes three steps: prepare for spread; establish an aim for spread; and develop, execute, and refine a spread plan. A secondary objective of the CDC is to engage all Canadians and to provide dying Canadians and their families with the necessary knowledge to advocate for appropriate change.

\section{Approach}

This initiative consists of two stages. Engagement, building consensus, and the development of recommendations comprise the first stage. A change management plan however is required to ensure uptake of recommendations arising from the first phase.

CDCs require public engagement. Intuitively the validity and representativeness of resulting recommendations require an appropriate depth and breadth of the consultation process. We propose a three-stage model intended to optimize the number, selection, and preparation of citizens.

Although 12 citizens are typically described, ideally it is intuitive that engaging more is better. Demographic representation is challenging when age, education, and geography play such important roles. As the number of citizens engaged however increase, the need to identify and synthesize input becomes more difficult, especially when considering qualitative data.

Selection of citizens is an important consideration. Random selection criteria, for example, are used in gathering input from public polls. This method is appropriate when the issues are not complex and easily understood. Discussion of physician assisted dying has moreover polarized the public and increased the probability of introducing bias if selection criteria are not monitored carefully. In particular, personal experience with death of a loved one will need to be considered. Cross-examination of potential jurors is required in complex and controversial legal cases. The need for clear criteria and management of costs are factors in citizen selection.

Finally, preparation of citizens is classically described as a two weekend process. Longer and more intensive education are offset by time and costs. Preparation can also be offset through the selection of appropriate jurors.

We propose two stages and the following steps which take into account these considerations.

\section{Stage I: Engagement and identification of best practices}

\section{Phase 1}

Quantitative and qualitative survey. The development of an online survey is to collect quantitative data. These methods allow for a large number of citizens to provide feedback. This phase was conducted by IPSOS (see Roulstan, this issue). Polls are anonymous which encourage truthful feedback. A final question will be provided asking interested participants to participate in a qualitative survey.

Analysis of qualitative data requires greater analytic resources and thereby limits the number of participants. A quantitative survey therefore serves as a filter to select knowledgeable and interested individuals. A qualitative survey probes more deeply and identifies broad feedback. Again, this stage can be used to vet candidates for participation in a CDC. Participation in both quantitative and qualitative survey processes requires sufficient education and interest in the topic. Participation will also stimulate discussions and selfeducation. Again, a final question asking interested participants for willingness to participate in a CDC will be included.

\section{Phase 2}

CDC proper. Selection of citizens from the nomination of interested individuals will ensure a more representative and better informed pool of candidates. A synthesis of data gathered by IPSOS and the scientific panel provide the basis for the CDC. A background article, plain language version of the evidence summaries, and a series of questions guide a dialogue between a citizen and scientific panel. The dialogue takes the form of a jury trial over several days, with a judge represented by the lay-panel facilitator. Including the chair, 12 members of a citizen panel required two preparatory conference calls and review of documentation. A resulting consensus statement was produced on the final day of the conference making reference to the deliberations, recommendations, and consensus statement. The statement can be found in its entirety at www.palliativecarematters.ca

Grundhal $^{7}$ describes in detail a process and roles for key players, including suggested committee and panel sizes.

\section{Manager (and administrative assistant).}

“• Formulating proposals to put before the steering committee.

- Recruiting the lay-panel facilitator.

- Managing the hearing of interested parties. 
- Advertising for, and short-listing, the lay-panel members and replying to all correspondence.

- Contacting potential scientific experts and following up the final selection once the program has been established.

- Managing the project finances.

- Dealing with the media.

- Providing practical assistance in the production of the final document.

- Preparation and publication of the final document.

- Ensuring that the final document is disseminated and arranging follow-up, such as written material or organizing debates.

In addition, the project manager coordinates meetings and takes minutes; deals with letters and telephone enquiries; distributes material; books accommodation; checks technical equipment for the conference (microphones, etc.); advertises the conference; and delegates practical tasks. A constant service needs to be supplied to the lay panel, the steering committee, and the facilitator."

Steering committee (6 individuals). At its first meeting the steering committee discusses the project from a general point of view. The project manager must communicate the motivation for selecting a consensus conference as a method for this project, the basic concept of the process, roles, strengths, and weaknesses, etc.

The role of the steering committee should be made especially clear. The time schedule will be presented by the project manager for approval. A list of interested parties in the field must be established. In addition, the steering committee must be informed of the Secretariat's selection of the lay-panel facilitator.

Finally, the steering committee sets up the framework of the introductory material, which will familiarize the lay panel with the topic of the conference. Such a briefing article may be compiled by the project manager on the basis of existing literature or it may be produced (externally) in the form of a separate article of 15-20 pages by a journalist or another appropriate person. The article will present the subject in general terms and describe the status, development, trends, attitudes, conflicts, etc. in the field. The writing must be unbiased: controversial attitudes should only be reportedobjectivity is the aim.

When the introductory material is ready, it is approved by the steering committee (possibly by correspondence rather than a meeting) before it is distributed to the lay panel.

\section{Lay-panel facilitator (like a judge in a jury trial).}

“• Making lay people feel comfortable and persuading them to work together.

- Managing the preparatory weekends, including the work of formulating the key questions.

- Chairing and managing the conference.

- Assisting and steering the lay panel's production of the final document.

- Focusing the lay panel's attention on key questions to be answered (so that conclusions in the final document are relevant to the debate and not on aspects that have not been discussed at the conference).

- Working closely with the project manager throughout the process." 7

\section{Scientific panel (6 individuals).}

- Being abreast of the latest knowledge. Having a good overview of the topic.

- Having good communication skills.

- Being receptive in debates.

\section{Lay panel (10-14 individuals). ${ }^{7}$}

- Gain some basic knowledge of the subject in advance by reading the introductory material and participating in the preparatory weekends.

- Draw up the key and sub-questions of the conference.

- Formulate a proposal for the composition of the scientific panel on the basis of their own discussions and the comprehensive list of scientific experts prepared by the steering committee.

- Question the scientific panel at the conference.

- Evaluate the received information and agree on attitudes and any recommendations in relation to the key questions of the conference on the basis of the presentations by the scientific panel and the debate at the conference.

- Write the final document of the conference.

Audience. A high profile lay facilitator supported by a communication strategy can be expected to draw an audience. An appropriate venue and process are required to accommodate the audience.

\section{Media and dissemination strategy.}

- printed reports

- journal articles

- stakeholder advisories

- press conferences

- news releases

- TV/radio broadcasts

- video products

\section{Phase 3}

The consensus statement is at the heart of a road map for Canada. The Conference Board of Canada was commissioned to write a report outlining potential policy options and implementation plans to guide governments, healthcare organizations, professionals, and other stakeholders. This step is an important step in the policy development cycle. A formal roadmap and action plan would be the next logical steps in this journey.

\section{Framework to Guide Selection of Topics and Formation of Questions}

During the last two decades, numerous organizations have engaged Canadians on the topic of palliative and end-of-life care. Table 1 lists the reports which contain, in whole or in part, recommendations to improve the quality of care received by dying Canadians and their families. Only organizations/reports which are national in scope have been included. Advance care planning is included while topics related to withholding and withdrawal of life-sustaining treatment, plus medical assistance in dying, are excluded.

A comprehensive review resulted in the identification of the 19 Canadian reports that identified a total of 192 unique recommendations on palliative and end-of-life care. Together, 


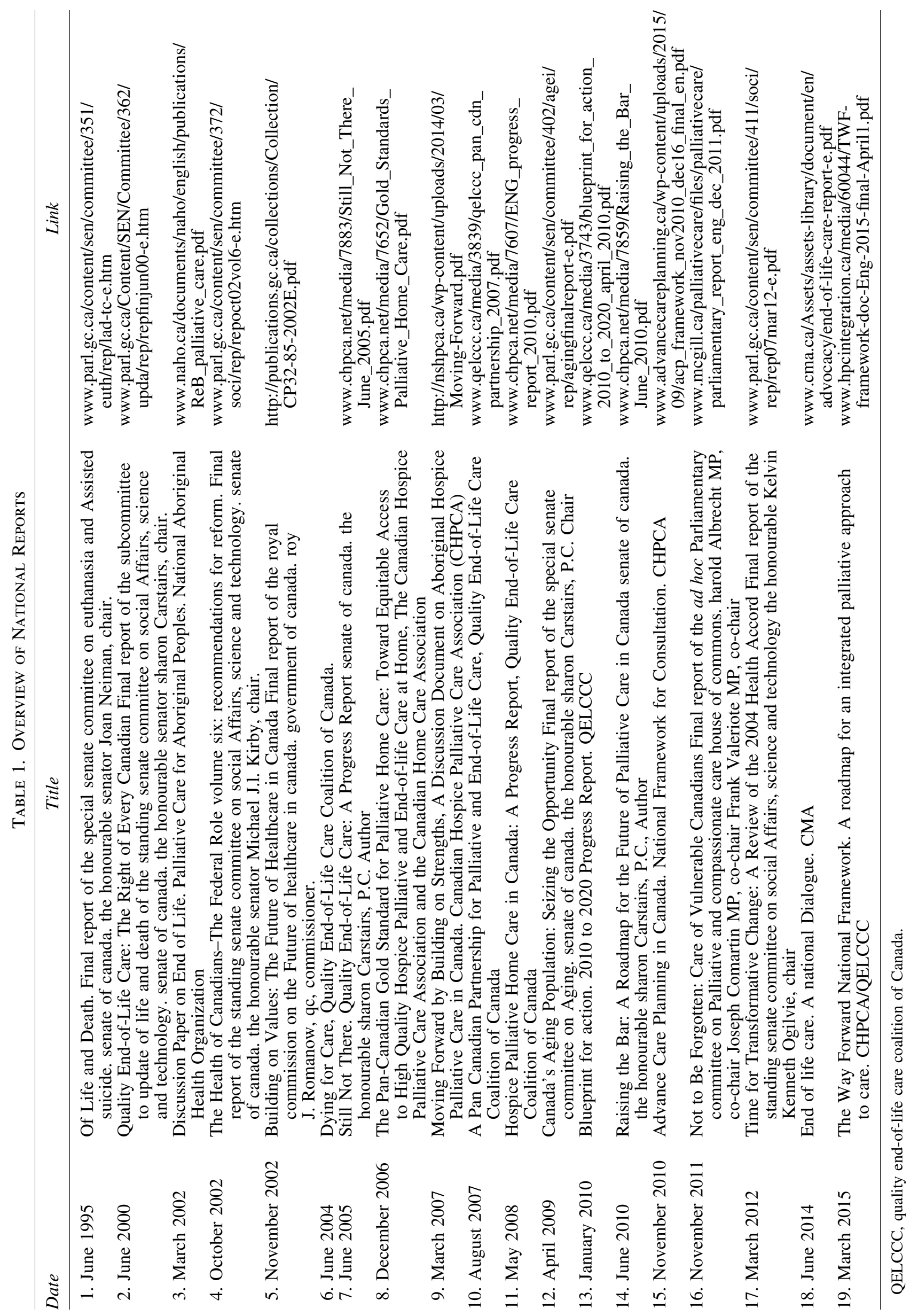




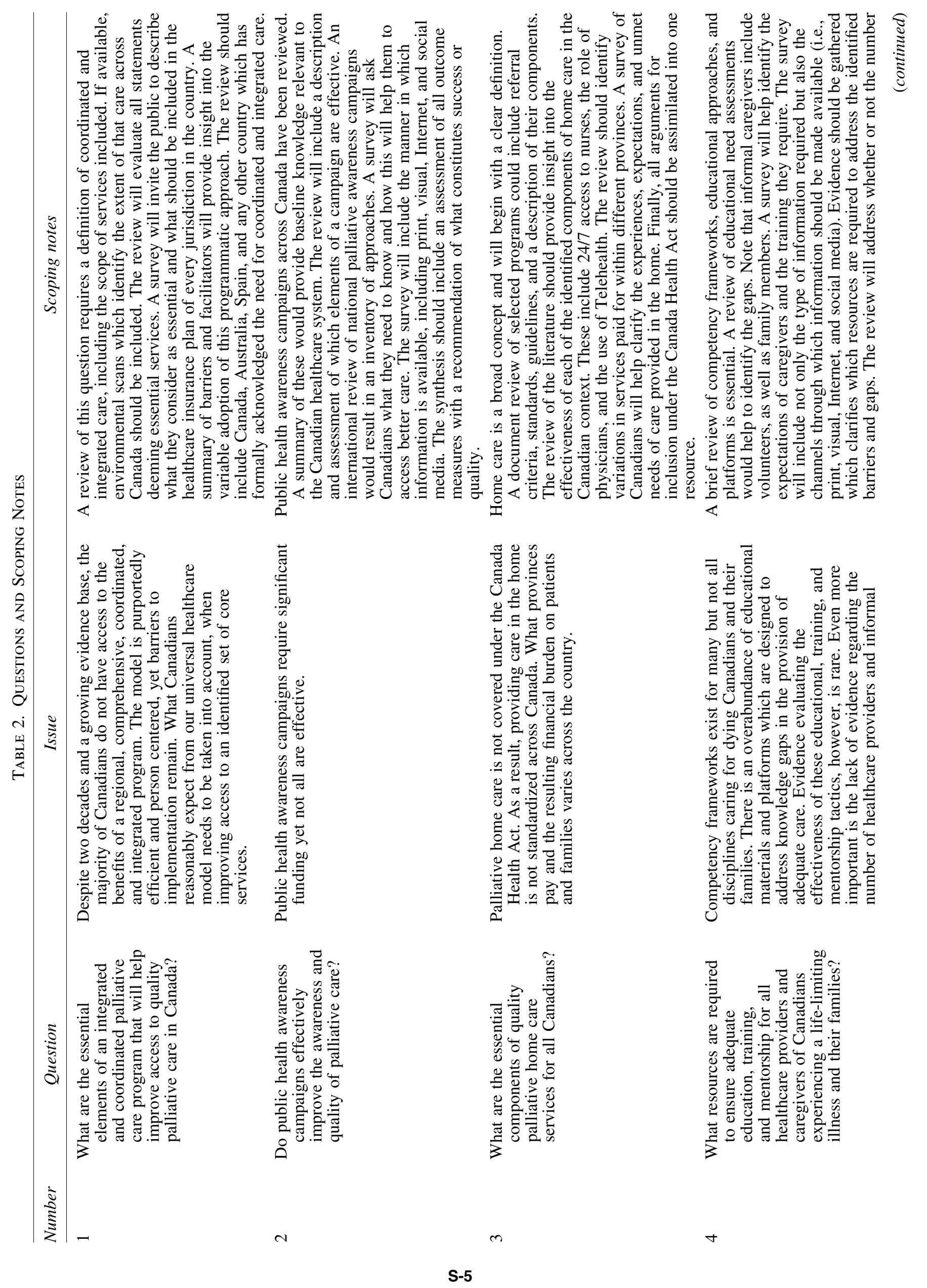




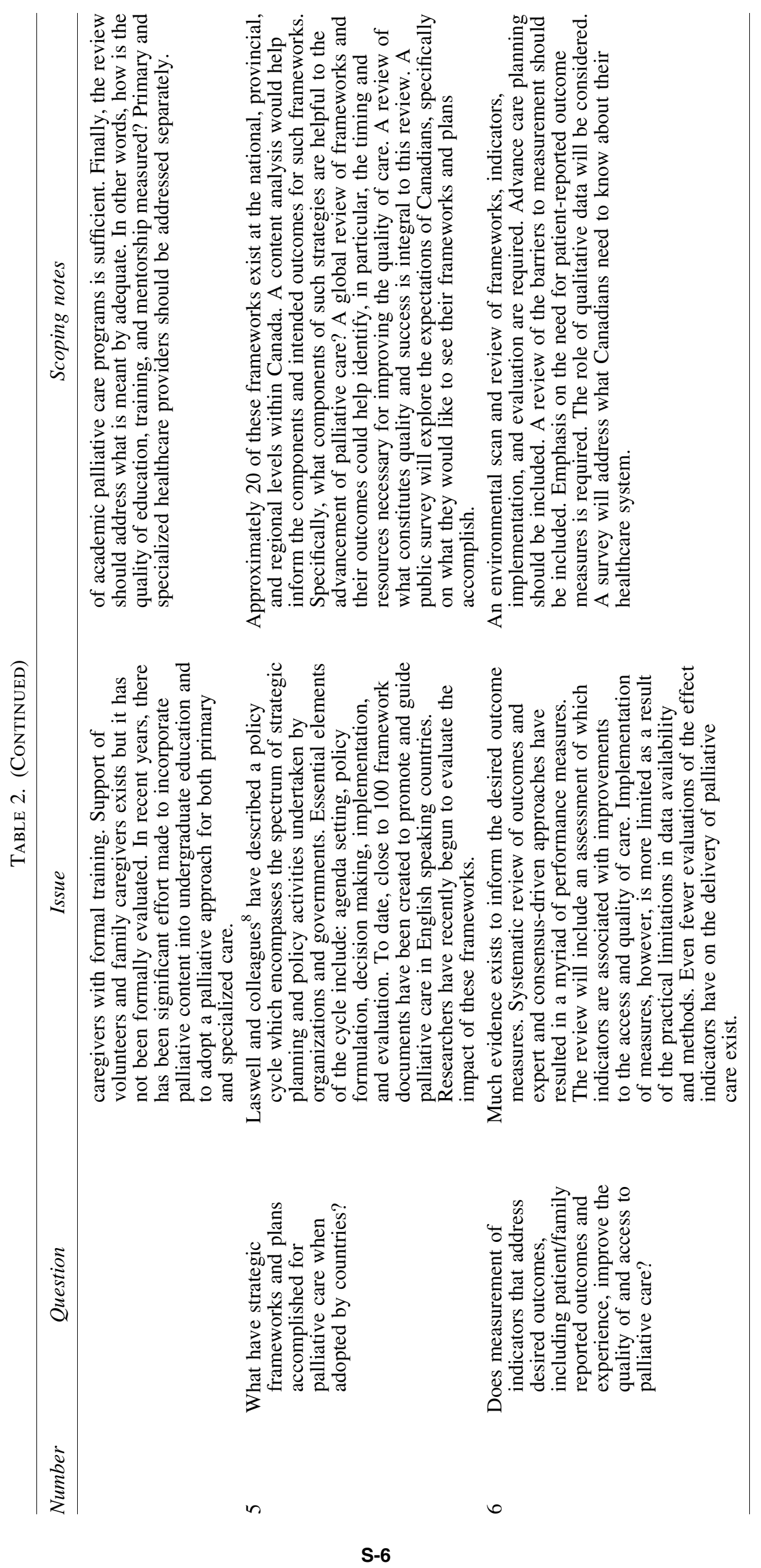


these reports and recommendations represent a vast consultation with Canadians. We wish to honor all the individuals and organizations by acknowledging and building on work that they have already completed. Not all recommendations, however, are relevant. Many are duplicated, others are dated and most importantly, many have already been implemented.

\section{Guiding principles}

The following principles apply to all questions.

1. Questions should generate evidence that is person centered. Patients and/or families must be included in the process at every level, contributing to design and delivery and providing ongoing feedback about how well the system is working.

2. Questions are applicable to all Canadians. When possible, evidence supporting the following populations of interest should be addressed: rural, remote, and indigenous.

3. Quality of palliative care includes the six Institute of Medicine dimensions: safe, effective, efficient, timely, person centered, and equitable.

4. Definition of Palliative Care (Adapted from A Model to Guide Hospice Palliative Care (C) Canadian Hospice Palliative Care Association, Ottawa, Canada, 2002.)

Palliative care strives to help patients and families:

- address physical, psychological, social, spiritual, and practical issues and their associated expectations, needs, hopes, and fears

- prepare for and manage self-determined life closure and the dying process

- cope with loss and grief during the illness and bereavement

Palliative care aims to:

- treat all active issues

- prevent new issues from occurring

- promote opportunities for meaningful and valuable experiences, personal and spiritual growth, and selfactualization.

Palliative care is appropriate for any patient and/or family living with, or at risk of developing, a life-threatening illness due to any diagnosis, with any prognosis, regardless of age, and at any time they have unmet expectations and/or needs, and are prepared to accept care.

Palliative care may complement and enhance diseasemodifying therapy or it may become the total focus of care.

Palliative care is most effectively delivered by an interdisciplinary team of healthcare providers who are both knowledgeable and skilled in all aspects of the caring process related to their discipline of practice. These providers are typically trained by schools or organizations that are governed by educational standards. Once licensed, providers are accountable to standards of professional conduct that are set by licensing bodies and/or professional associations.

\section{Canadian Reference Working Group}

A Canadian Reference Working Group (CRWG), representing the Canadian public and major stakeholders, was convened on June 15, 2016, and a nominal group technique was used to facilitate the meeting to choose the recommen- dations which have the greatest likelihood to improve the quality of palliative care for all Canadians. The CRWG reached consensus on the identification and wording of six questions. A face-to-face meeting was followed by four rounds of editing to arrive at the final questions and scoping notes. A committee of 16 represented a balance of expert, stakeholder, and public representatives. The questions are designed to help gather the evidence which addresses significant national recommendations. Evidence will be gathered from qualitative and quantitative inquiry of public alongside scientific expert review of the academic and gray literature. Where practical, we will ask for stakeholder insights into an analysis of the current state.

All questions align with one or more recommendations from national reports on palliative care. Nominal group technique was used to elicit those recommendations which were deemed to have greatest impact. The content and order of the six questions therefore reflect the priorities of the CRWG. The questions struck an important balance in scopeneither too narrow to be useful nor too broad to weigh down the evidence gathering process. A description of the issues and scoping notes is meant to develop a common language and understanding of why the questions are important. These also serve to guide a scientific panel tasked with collection of evidence addressing the respective questions. The final questions and scoping notes are provided in Table 2.

\section{Stage II: Change management, policy implementation, spread}

Financing and delivery of healthcare fall within provincial jurisdiction. A report summarizing the recommendations informed by scientific experts and citizens is a powerful and necessary first step. Gaining the attention of health ministers, establishing the urgency for change, sequestering sufficient resources, and developing appropriate policy and procedures will require a plan. Three steps are contemplated.

Step 1: Prepare for spread

Step 2: Establish an aim for spread

Step 3: Develop, execute, and refine a spread plan

\section{Author Disclosure Statement}

No competing financial interests exist.

\section{References}

1. Scherstén T: Topic and scope of consensus development conferences: Criteria and approach for selection of topics and properties for assessment. In: Goodman C, Baratz SR (eds): Improving Consensus Development for Health Technology Assessment: An International Perspective. Washington, D.C.: National Academy Press, 1990.

2. Committee of the Institute of Medicine Council on Health Care Technology. Consensus Development at the NIH: Improving the Program. National Academy Press, Washington, DC. 1990.

3. Fink A, Kosecoff J, Chassin M, Brook RH: Consensus methods: Characteristics and guidelines for use. Am J Public Health 1984;74:979-983.

4. Oliver MF: Consensus or nonsensus conferences on coronary heart disease. Lancet 1985;1:1087-1089.

5. Lomas J: The consensus process and evidence dissemination. Can Med Assoc J 1986;134:1340-1341. 
6. Massoud MR, Nielsen GA, Nolan K, et al.: A Framework for Spread: From Local Improvements to System-Wide Change. IHI Innovation Series White Paper. Cambridge, MA: Institute for Healthcare Improvement, 2006. www.IHI.org (last accessed June 26, 2017).

7. Grundhal J: The Danish consensus conference model. Public participation in science: The role of consensus conferences in Europe 1995 (pp. 31-40). London: Science Museum.

8. Lasswell HD: The policy orientation. In: Lemer D and Lasswell HD (eds.), The Policy Sciences. Stanford University Press, Stanford, CA, 1951.
Address correspondence to: Konrad Fassbender, MA, PhD

Department of Oncology University of Alberta 3rd Floor, Brain and Aging Research Building 8710112 Street

Edmonton, Alberta T6G $2 M 8$

Canada

E-mail: konrad.fassbender@ualberta.ca 